\title{
Ubiquitous Simultaneity: A Design Workflow for an Information Rich Environment
}

\author{
JASON S. JOHNSON \& MATTHEW PARKER \\ University of Calgary
}

In June of $\mathbf{2 0 1 4}$ over $\mathbf{1 7 0 0}$ entries were submitted to a competition for a proposed Guggenheim Museum in the Finnish capital of Helsinki. Winners had no guarantee that the project would be built, yet architects from around the world spent huge amounts of capital and time to produce what was described by the Director of the Solomon R. Guggenheim Foundation as "an unprecedented volume of design information that is now freely available for study and use". Just like that the intellectual capital of nearly two thousand architects was distributed essentially free of charge to the public. This represented a massive upload of the architectural intentions and pre-occupations of a generation of architects that was not used or evaluated in any way other than to promote the competition itself. This paper focuses on the development of a design process that makes use of large repositories of previously designed projects as the content for a new kind of design workflow. The leveraging of computational techniques and large data rich territories is described and its preliminary outputs presented.

\section{INTRODUCTION}

In 2002 the Lower Manhattan Development Corporation launched the World Trade Center Site Memorial Competition and received over 2000 entries. In 2014 more than 1000 proposals were submitted for the design of the Bamiyan Culture Centre Design Competition solicited by UNESCO and the Ministry of Information and Culture of Afghanistan. Later that same year the Solomon R. Guggenheim Foundation launched the Guggenheim Helsinki Design Competition and received more than 1700 entries. The architectural outputs for these competitions required a series of architectural drawings (plans, sections and elevations) and a selection of diagrams describing the projects' circulation and environmental strategies and at least one interior and one exterior rendering. With no guarantee the winning project would be constructed architects eagerly donated their time and expertise for nothing more than the potential bragging rights. Motivated by the outside chance that the winning entry might be constructed, architects essentially transferred their intellectual property to organizations that immediately made them available for comment (positive and otherwise) by anyone with access to the internet. While the finalists and a few other projects received some measure of attention and publicity, the vast majority of submissions were largely forgotten, architectural debris swept under the rug of a contemporary design culture constantly searching for the next best thing. These architectural repositories are rarely sorted within any sort of order or intention (only the short listed candidates are called out and placed in their own gallery) and often result in a content catalog often so extensive that the value of any one project is lost within the ever expanding repository of architectural images. The proliferation of architectural outputs through competitions combined with the commitment of various digital platforms to store and disseminate these outputs feeds into a condition previously described as ubiquitous simultaneity (Johnson, Jason S, Vermillion 2016) in which ever expanding and updated data rich catalogs of information have become available in real time and mapped across spatial interfaces. We contend that ubiquitous simultaneity challenges the discipline of architecture to address the changing role of the architect within an information rich environment oversaturated with representational images of architectural production. In order to operate within this condition, we have proposed a workflow that relies on computer vision to search and organize large datasets of architectural outputs, combined with a design strategy of heteromorphic deformation to mobilize SIFT algorithms towards the embedding of speculative historical design artifacts into newly formed architectural proposals.

FROM 3D TO 2D: THE USE OF OPTICAL PROSTHETICS IN ARCHITECTURE

Central to this workflow is the use of computer vision and its reliance on a class of SIFT algorithms to enable a level of inhuman vision capable of 'seeing' despite a lack of eyeballs, rods, cones and visual cortex. SIFT (Scale-Invariant-Feature-Transform) algorithms enable computer vision to identify specific image features that are invariant to scaling, rotation, changes in illumination and 3D camera viewpoint. Extracted from each pixel of an input image and encoded with contextual 


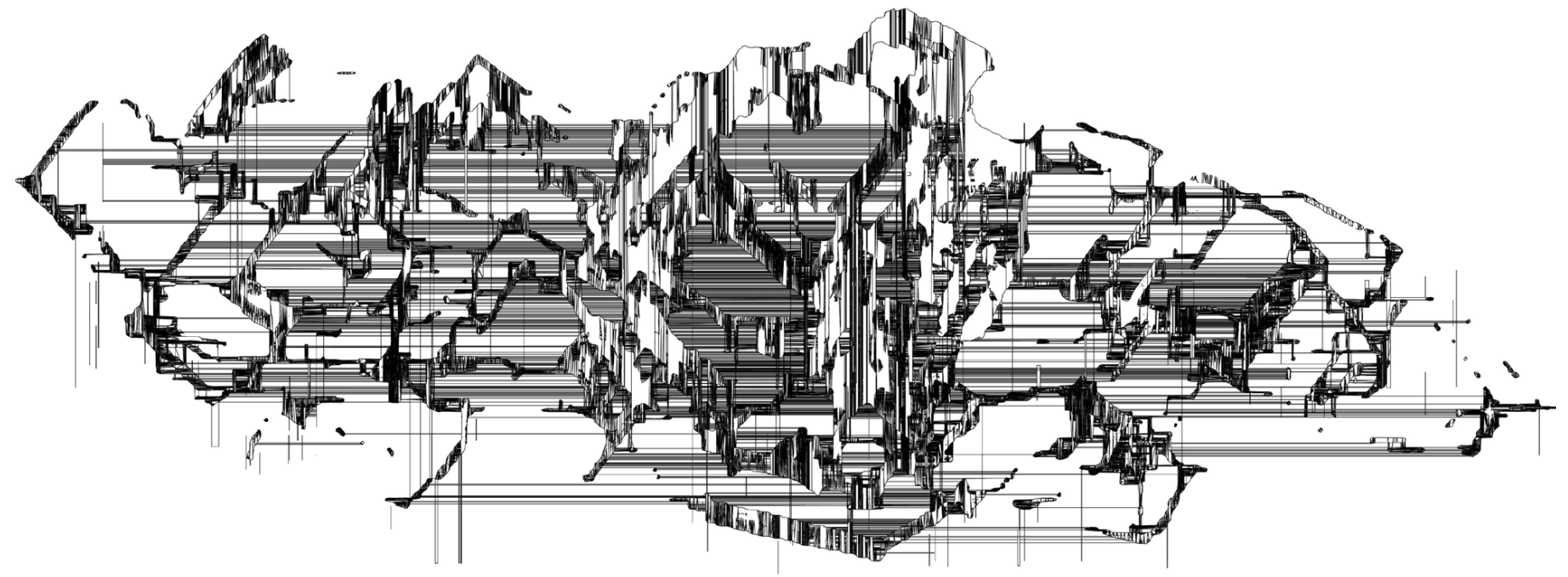

Figure 1: Aggregate Section Image created through SIFT'ing multiple project cross sections without biasing or post-production.

information through a process that reduces images to a large number of highly distinctive keypoints, SIFT algorithms are deployed for the purposes of image retrieval, image stitching, object recognition, gesture recognition, match moving and the digital reconstruction of virtual environments from a collection of two-dimensional image data.

Once a series of images has been codified through SIFT processing, computer vision algorithms search image datasets for correlate keypoints, which are superimposed and mapped on top of each other, flattening multiple datasets into what we refer to as a data-rich-territory; a datascape whose quantity of data trees does not change but the complexity of each data tree is magnified to respond to the superimposition of multiple bodies of soft data. At their most basic level, SIFTs abstract two-dimensional digital images to their geometric compositions for the purposes of matching across dynamic image sets. Within this workflow we deploy SIFTs in two ways; on the one hand we exploit SIFTs for their ability to recognize, identify, codify and sort the extensive datasets of outputs generated through platforms like architectural competitions. On the other hand, we mobilize new bodies of data generated through the SIFT processing of large datasets (similar to those produced through architectural competitions) in an attempt to recycle architectural intention towards the production of new and novel design outputs.

The notion that architecture relies on machines, in this case computer vision, to produce new data-intensities towards its own poesies is not new, in fact it can be situated within a historic lineage dating back to the tenth century and the first appearance of Alhazen's camera obscura, through the increasing number of visual prostheses (lenses, astronomic telescopes, etc.) of the Renaissance. These devices confront objects in a fundamentally inhuman manner that exposes excess data intensities otherwise concealed from unmediated human perception. But why does architecture rely on said devices to augment our perception of the surrounding context? Traditionally the use of optical prosthetics in architecture stems from a desire to produce accuracy and legibility among the representations necessary for architecture's visualization. Plan, section and elevation drawings are common to all architectural projects, despite the fact that users are incapable of experiencing an actual building in this manner. These conventions remain consistent within architecture due to the level of accuracy and legibility they are able to communicate, a level of fidelity that results from their 2-dimensional nature. However, we experience the context(s) of architecture through $\mathrm{n}$-dimensional environments that require mediation to accurately produce the architectural outputs necessary for its construction. It is to this point that architecture maintains a relationship with optical prostheses such as Alberti's veil, or the use of photograph to flatten the spatial and temporal dimensions of our context so to more accurately design and construct architecture.

The use of optical prosthetics within architecture has resulted in a homogenous environment of design conception and visualization - the world of the image. Within this world architectures' image is produced and reproduced at an unfathomable rate, and while our workflow exploits SIFTs and computer vision to sort and make sense of these extensive datasets this paper would like to focus on the ability of computer vision and SIFT algorithms to invert architecture's relationship to optical prostheses by extracting $n$-dimensional vectors from the data contained within existing and historic 2-dimensional architectural outputs towards the production of new architectural intentions.

\section{PRODUCING NEW CONTENT: FROM 2D TO 3D}

The process of converting digital images into usable data within SIFT workflows converts an image into soft data capable of transforming and responding to adjacent datasets without losing its autonomy. To put this another way, SIFT algorithms multiply and animate existing 


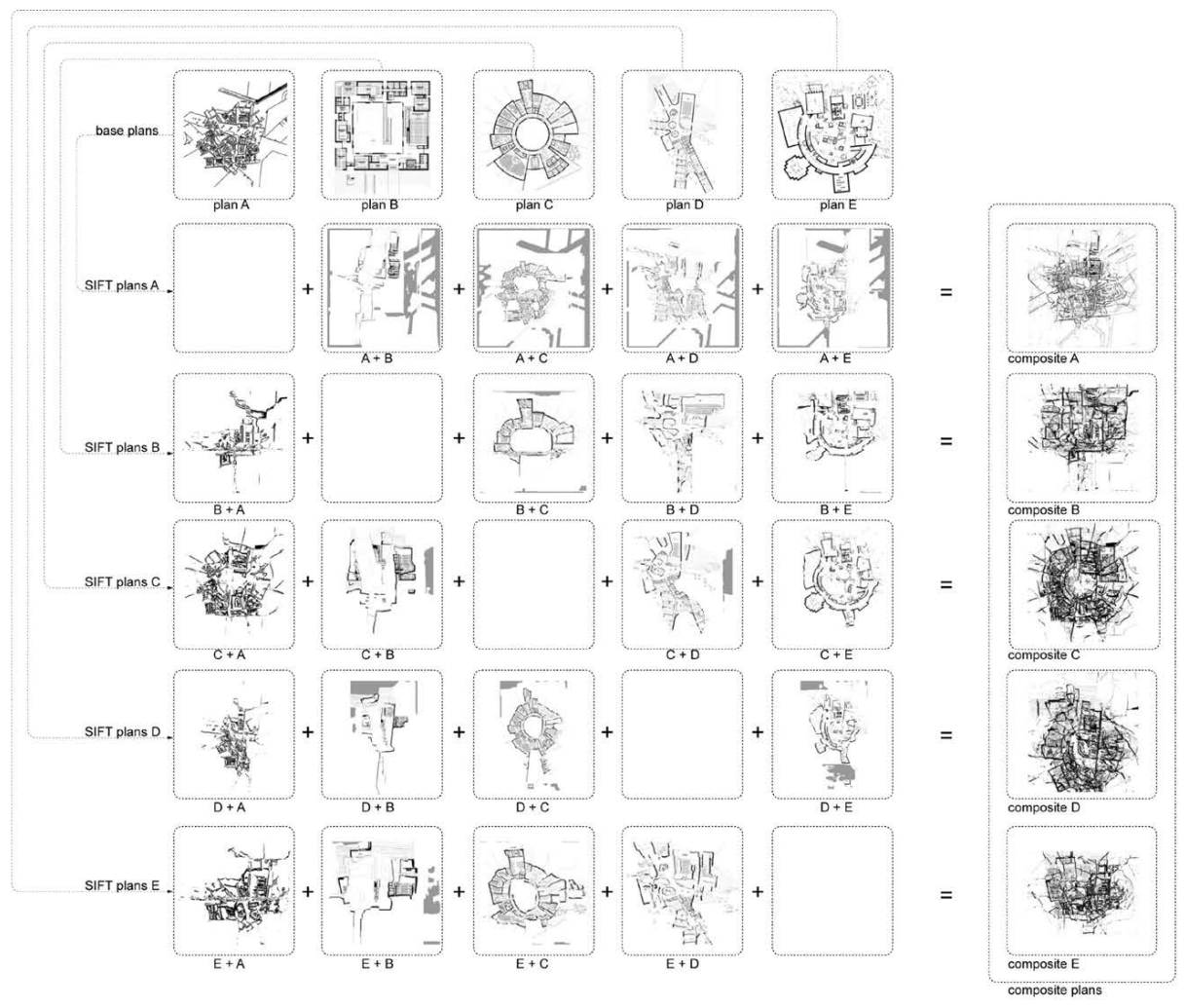

Figure 2: SIFT composite image diagram. Composite plans created through the combination of various plan types found in the image data set.

images through the production of new data. Easily overlooked within this discussion that explores the ability of SIFT algorithms to produce new content, is that computer vision relies on images of existing artifacts to produce its vision. The implications of this is that anything 'new' produced through computer vision exists in relation to a set of input images and visual data that describes a previously documented artifact. This is not to say that a workflow built around Computer Vision and SIFT algorithms cannot produce novelty, in fact the opposite is true; SIFTs must produce 'new' to signify the existing. Just as an artist produces novelty through the intentional augmentation of a medium, computer vision manufactures digitally curated images through an internal and autonomous interpretation of data.

This workflow processes large aggregate sets of architectural drawings (plans and sections) by abstracting recognizable architectural characteristics to their geometric compositions and assigning them codified SIFT keypoints. SIFT'd plans and sections (Figure 1) are processed and superimposed (Figure 2) constructing $\mathrm{n}$-dimensional vectors that are extracted as a vector-flow-range, a spreadsheet that includes the UV values for each pixel's vector, and the maximum and minimum vector difference between correlated images. These vector values are mapped to 3D model space by extracting the UV directional values of a pixel and its associated vector magnitude to produce dynamic vector-flow-fields, that represent the amount of movement a keypoint undergoes as correlated keypoints are composited.

The vector-flow-fields produced through the processing of plan drawings are mapped to the $X Y$ plane of $3 D$ model space with the vector-flow-fields associated with sectional drawings mapped to the $X Z$ and $Y Z$ planes (dependent on their longitudinal or latitudinal qualities) creating a 3-dimensional vector field (Figure 3). By testing plan based and section based vector-flow-fields for intersection new geometries begin to take shape. Mesh faces are produced around the point of intersection with the face extruded outwards perpendicular to the plane of the dominate vector. The faces act as a tracing of complexity contained within and across correlated keypoints, with the overall 'complexity' of the output image defined by the number of input images and the number of overlapping and intersecting SIFT descriptors inherent to a composite set of architectural drawings.

\section{THE IMPORTANCE OF BIAS}

Intrinsic within processes that deploy aggregation techniques is the need to give weight to the various pieces of data present in the composited information bodies. The first approach used was to give all the images the same weight in order to observe the areas of greatest commonality in the larger data sets. This approach has been used by the visual artist Jason Salavon and others to produce almost ghostly images made up of hundreds of instances of similar photographs. While this technique is very good at describing tendencies within a large image set, it does not allow the designer to influence the formal qualities of the outputs, outside of the initial image selection.

In order to allow for the production of 2 dimensional plan and section images that take on distinct and useful, in other words desired features, it was necessary to introduce a technique for biasing specific plans or sections within the overall image set. First in this process was the identification of outliers within the plan and section sets. These were images which most deviated from the "typical" plan 


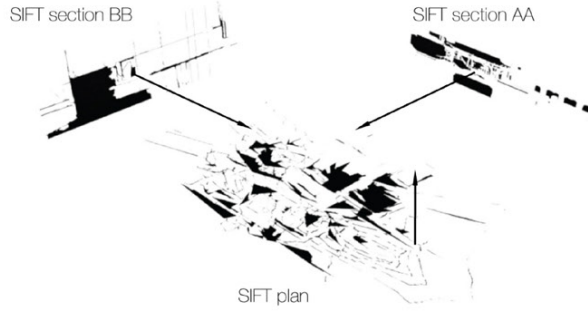

soft bodies rendered as architectural drawings

are oriented relative to their orthographic perspectives

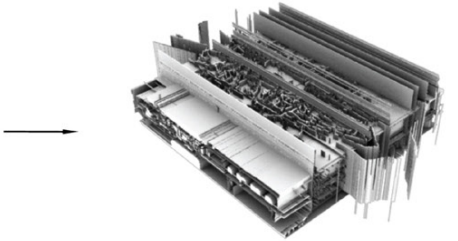

SIFT Keypoints within bodies of soft data are extruded along $n$-dimensional vectors

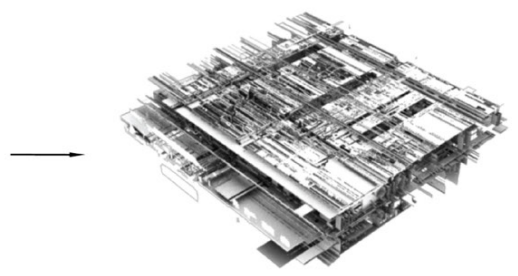

SIFT bodies are intersected and meshed to produce three dimensional data-rich teritories
Figure 3: 2D to 3D workflow diagram for SIFT geometries

organizations of the submissions. The second step was to choose "winners" or organizational structures that were most desirable to the designers. Biasing or playing towards tendencies is achieved by applying weighting to specific images in the set and running the SIFT workflow. These results were then analyzed and the best options processed as described in Figure 3.

Finally a set of 2D plans and sections are produced through a process of removing "orphaned" or disconnected 2D vectors in the resulting images in a vector based image editing software. These images (Figure 4) form the basis for the $\mathrm{n}$-dimensional modeling workflow (Figure 3).

\section{THE PROPOSAL}

The Museum of Recycled Intention is a project designed by implementing this workflow on a dataset that included all of the finalist designs from the competition jury and those selected by editors at various design aggregation websites. This selection set offered an opportunity to incorporate or "recycle" projects that had already been weighted by a pool of experts. The result of this project is a series of interarticulated spatial sequences that embed horizontal and vertical structural systems within a series of diagonal voids (Figures $4,5)$. The galleries are arranged in horizontal bands through the structure broken up by several large scale voids. These voids are not surprising given the fact that most proposals for the museum competition made use of large multi-storey gallery spaces surrounded by smaller scale galleries and ramps. The process of producing $n$-dimensional geometry within the workflow perhaps predictably produces horizontally directional articulation within the surfaces of the main geometries. This articulation provides a framework for material distributions throughout the interiors of the project.

\section{CONCLUSION}

A design process that leverages large amounts of existing data, pre formatted to comply with the representational standards of a profession, has the potential to change the ways in which we view architectural "wastage". Generative design spaces of many types are already producing highly immersive, customized and programmatically varied environments based on algorithmic processes that mine existing formal qualities of natural and urban systems as their base design parameters. One needs look no farther than the random world generators in Minecraft to visualize the range of performance that such a system allows for. In this instance we are proposing that the vast quantities of data that are being produced for both built and unbuilt projects, represent a resource that can be mined for specific characteristics and used as content for a workflow made up of form finding algorithms. This content could become increasingly embedded with meta data that helps us understand the intentions of the original designs. What are the prevalent circulation patterns within specific building typologies? What are the most successful formal strategies in relation to a range of programmatic requirements? What are proportional tendencies within projects that succeed in competitions or perhaps more importantly in post occupancy analysis?

This project proposal represents a first step in the direction of trying to produce a parametricism that departs an explicit relationship with well-established formal qualities of highly articulated components arrayed on smooth sub-surfaces towards a deeper form of the interarticulation that Schumacher has argued for over the last decade. This approach offers the potential for an architectural project that learns from existing and speculative projects in way that was not fully possible in the past.

\section{ENDNOTES}

1. If one is to apply the calculator of architectural desperation proposed by Mark Foster Gage (2014) to all three of these competitions, we get a conservative estimate of over 169 million dollars of architectural donations.

2. For a detailed explanation of SIFT algorithms and how the breakdown and process visual data contained within two-dimensional images see Johnson and Parker This Is Not a Glitch: Algorithms and Anomalies in Google Architecture (2014).

3. Soft data is term put forward by this research to describe data set that possesses the ability to elastically deform in response to external forces while not altering its original unique characteristics. Within this research $\mathrm{AO}$ composites multiple bodies of soft data into single data-rich territories.

4. The ability to sort and organize through deploying SIFT workflows is covered in Architectural Heat Maps: A Workflow for Synthesizing Outliers, Historical and Speculative Data (2016) 


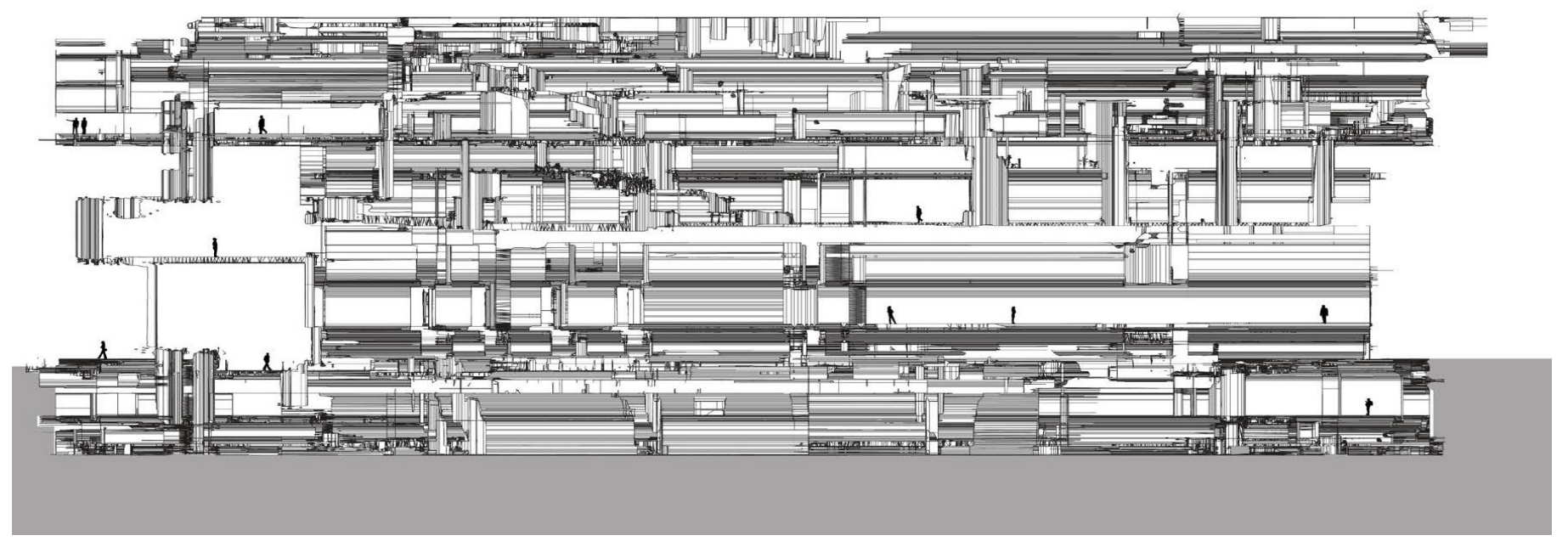

Figure 4: Drawing of SIFT geometries

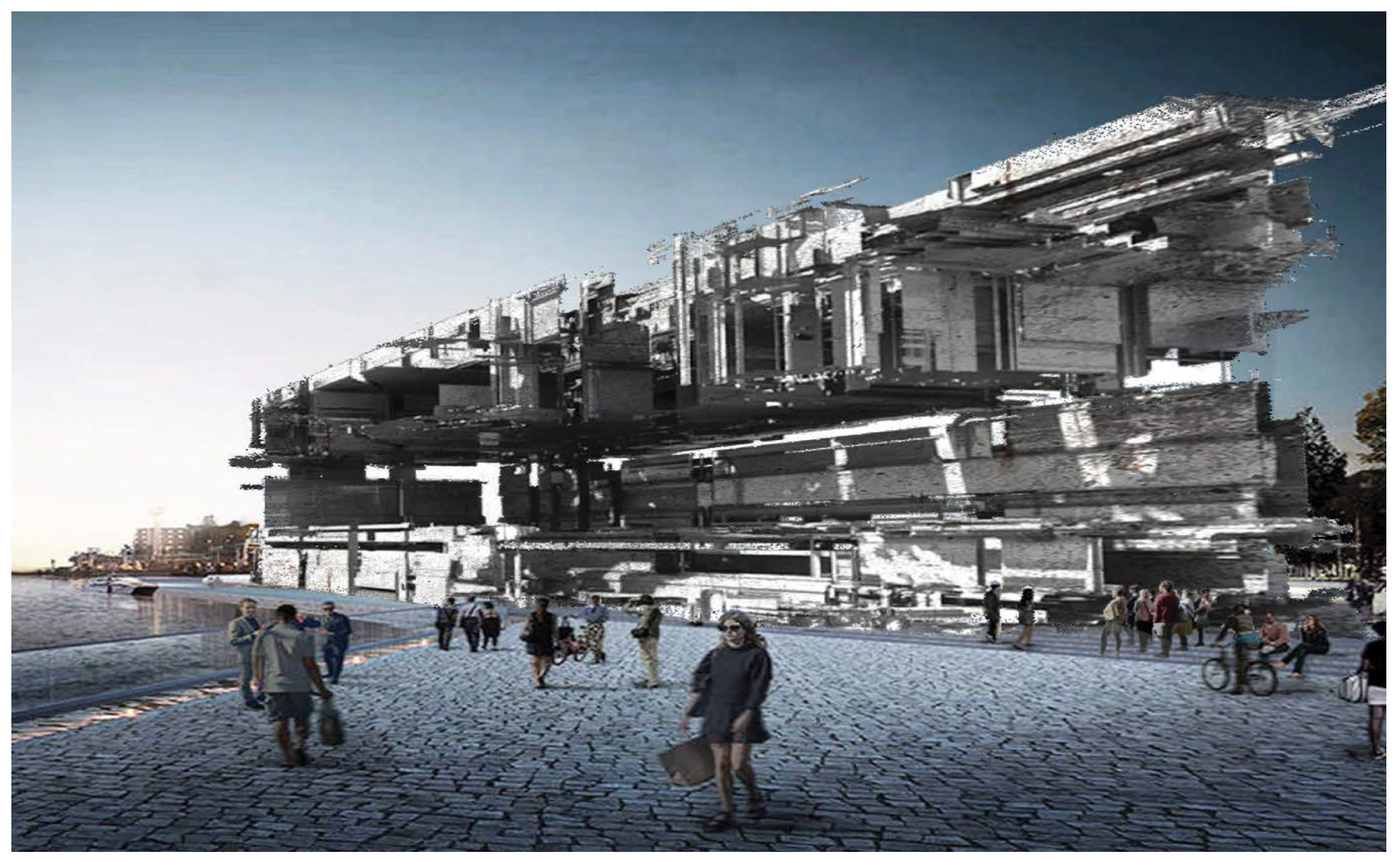

Figure 5: Drawing of SIFT geometries 
5. Johnson \& Parker: This Is Not a Glitch: Algorithms and Anomalies in Google Architecture (2014)

6. The dominate vector is determined by testing intersecting vectors and identifying the vector with the largest magnitude. Extrusion perpendicular to the plane of origin is utilized as extrusion along the length of the prevailing vector, or an averaging of all directional vectors, produced a general illegibility and fuzziness within the drawings.

\section{REFERENCES}

Gage, M. F., 2014. Rot Munching Architects. In Perspecta 47 The Yale Architectural Journal, edited by James Andrachuk, Chirstos C. Bolos, Avi Forman, Marcus A. Hooks.

Johnson, J., Parker, M. 2014. This Is Not a Glitch: Algorithms and Anomalies in Google Architecture. In Proceedings of the 34th Annual Conference of the Association for Computer Aided Design in Architecture, 389 - 398. Los Angeles, California.

Johnson, J., Parker, M. 2016 (forthcoming). Architectural Heat Maps: A Workflow for Synthesizing Outliers, Historical and Speculative Data. In Proceedings of the 36th Annual Conference of the Association for Computer Aided Design in Architecture, Ann Arbor, Michigan.

Johnson, Jason S, Vermillion, Joshua. 2016. "Exercises for Integrating Data and Form." In Digital Design Exercises for Architecture Students, edited by Joshua Johnson, Jason S, Vermillion, 1st ed., 195-238. New York: Routledge.

Lowe, David G. 1999. “Object Recognition from Local Scale-Invariant Features." In Proceedings of the Seventh IEEE International Conference on Computer Vision, 2:1150-1157. doi:10.1109/ICCV.1999.790410. http://ieeexplore.ieee.org/lpdocs/ epic03/wrapper.htm?arnumber $=790410$.

Lowe, David G. 2004. "Distinctive Image Features from Scale-Invariant Keypoints." International Journal of Computer Vision 60 (2): 91-110. doi:10.1023/B:VISI.0000029664.99615.94.

Schumacher, Patrik. 2008. "Parametricism as Style - Parametricist Manifesto." Darkside Club, 11th Venice Architecture Biennale: 1-5. http://www.patrikschumacher.com/Texts/Parametricism as Style.htm. 